\title{
Dual Stage Ytterbium Doped Fiber Laser in MOPA Configuration
}

\author{
M. Fernandez-Vallejo ${ }^{*}, 1$, D. Monelli ${ }^{2}$, D. Passaro ${ }^{2}$, A. Cucinotta ${ }^{2}$, S. Selleri ${ }^{2}$ and M. Lopez-Amo ${ }^{1}$ \\ ${ }^{I}$ Public University of Navarra, Department of Electric and Electronic Engineering, Campus de Arrosadia, Pamplona, \\ Spain \\ ${ }^{2}$ University of Parma, Viale G.P. Usberti 181/A Parma, Italy
}

\begin{abstract}
We report the experimental demonstration of an Ytterbium doped fiber laser (YDFL) composed by two stages in cascade. Two sorts of Ytterbium doped fiber (YDF) with different concentration have been used. A proper characterization of the YDFs has been performed in order to find the optimum YDF length. To this end, the output power and the slope-efficiency have been calculated and the signal amplification and the amplified spontaneous emission (ASE) spectra have been analyzed, which are strictly related to the absorption and emission cross-section of the YDF. The first stage has been studied for both continuous wave (CW) and pulsed regime. The results show that by using an YDF with a high slope-efficiency, up to $66 \%$, it is possible to reach an emitted peak power of $30 \mathrm{~W}$ in a pulsed regime.
\end{abstract}

Keywords: Ytterbium, Ytterbium doped fiber, optical fiber laser and amplifiers, slope-efficiency, Master Oscillator Power Amplifier (MOPA).

\section{INTRODUCTION}

Since the advent of the laser in 1960 at Bell Labs (California), one of the main challenges has been to increase its output power. The vast experience in the context of optical communication systems has influenced positively in the development of high power fiber lasers, speeding up their progress [1-4]. This kind of lasers is able to cover a wide range of applications: medical, monitoring and remote sensing, laser radar, space communications, materials processing and defence, among others [5]. However, high power lasers require some specific characteristics of the laser source, such as high beam quality and efficiency, which often are not straightforward to achieve.

At first, the field of high power lasers was dominated by conventional bulk solid state lasers. Nevertheless, advances in fiber laser technology have made possible the development of commercial fiber lasers, which launch similar power values to the traditional solid-state sources. Fiber lasers have noteworthy advantages over bulk lasers: they are more compact, more efficient, offer a better beam quality and a higher brightness. On top of that, the fiber has an excellent heat dissipation capability due to its geometry.

Initially, Neodymiun was considered as a possible dopant to develop high power fiber lasers. But, in a short period of time, it was found that the properties of Ytterbium were better than the Neodymiun ones. The most outstanding features of Ytterbium are: minimum quantum defect between the emission wavelength and pumping, thus the efficiency is very high; absence of Excited State Absorption (ESA); it is possible to dope fiber with high concentrations, as a result high gain is achieved with short length of fiber; simple spectroscopy scheme compared with other rare earths and a broad

*Address correspondence to this author at the Public University of Navarra, Department of Electric and Electronic Engineering, Campus de Arrosadía, Pamplona, Spain; Tel: +34 94816 6044; Fax: +34 94816 970; E-mail: Montserrat.fernandez@unavarra.es bandwidth of amplification [6,7]. The YDF provides an amplification bandwidth from $975 \mathrm{~nm}$ to $1200 \mathrm{~nm}$ roughly.

Certain constrains appear when high power fiber lasers are desired. Among those, the thermal effects in the fiber, which are not negligible when working with very high emitted power. Moreover, the power boundary is marked by the onset of nonlinear effects, such as Kerr effect, four wave mixing, Brillouin and Raman scattering, which appear due to the high power density in the core area [8,9]. To cope with these impairments, some fibers have been specifically designed in order to withstand high powers. In general, the main goal is to increase the area of the core and the fundamental mode while operating in single mode regime.

As mentioned above, conventional fibers used in communication systems pose a problem if they are used for high power lasers. This restriction can be overcome by using special fibers such as the double-clad fiber [10]. The pump power is gradually absorbed by the doped core along the entire fiber, as a result the output beam gives high brightness and power. The main disadvantage of double-clad fibers is that the overlap between the pump power and the doped core is small, hence the efficiency decreases. Different techniques are used to improve significantly the absorption of the pump $[11,12]$.

On the one hand, Large Mode Area (LMA) fibers are specially designed to be suitable for high power applications. They have relatively wide modal areas and it is possible to work in single mode regime or supporting only a few modes $[13,14]$. On the other hand, photonic crystal fibers (PCFs) [15] are an interesting alternative to single-mode LMA fibers. They have the great ability to be single mode in a very wide bandwidth [16,17]. Another advantage of the PCFs is the possibility of creating an air region which acts as a cladding to achieve a double-clad fiber [18].

Finally, to achieve high fiber laser power; a great number of experimental configurations are present in literature 


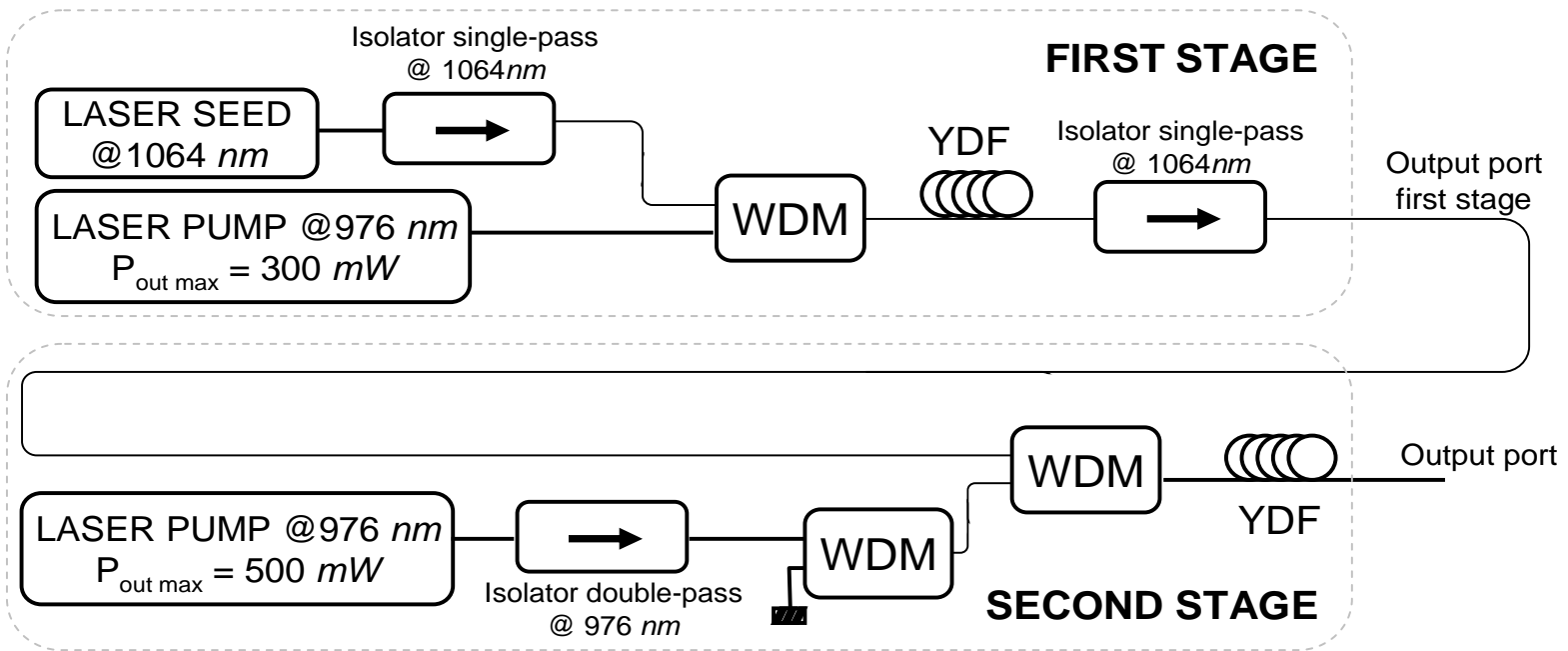

Fig. (1). Experimental set-up for the continuous wave (CW) Ytterbium doped fiber laser. WDM: wavelength division multiplexer. $\square$ : Index matched fiber end to suppress reflections. YDF: Ytterbium doped fiber.

$[19,20]$. A typical configuration is the so-called master oscillator power amplifier (MOPA) configuration. The master oscillator is a single-mode laser that is the seed laser of the power amplifier. The most significant advantage, in addition to its complete modular architecture, is that the generation of the pulse is totally independent of the optical amplification process because the characteristics of the modulation signal can be controlled electronically [5]. This capability is pivotal in some applications, such as materials processing, where pulses with duration of the order of nano or pico seconds are required.

\section{METHODOLOGY}

Fig. (1) shows the experimental setup for the proposed YDFL in CW regime. The scheme utilizes Corning HI 1060 fibers to connect the two different subsystems of the laser.

The first stage consists of two lasers: a seed laser by Lumics (LU1064M010) which works at $1064 \mathrm{~nm}$ and delivers $200 \mathrm{~mW}$ when it works in $\mathrm{CW}$ and a pump laser by EM4 (EM4 P162) with a center wavelength in the range of $976 \mathrm{~nm}$ to $980 \mathrm{~nm}$ which can emit up to $350 \mathrm{~mW}$. In order to combine both the signal at $1064 \mathrm{~nm}$ and the pump power at $976 \mathrm{~nm}$, a Wavelength Division Multiplexer filter (WDM) is placed before the single cladding YDF. Two single-pass isolators operating at $1064 \mathrm{~nm}$ are placed to avoid that undesirable reflections arrive to seed and pump laser.

The second stage includes a fused-silica WDM to combine the first stage output with the pump signal of the second one. The pump of the second stage emits a power up to 500 $\mathrm{mW}$ at the wavelength of $976 \mathrm{~nm}$. After the pump laser a double pass isolator at $976 \mathrm{~nm}$ and a WDM filter have been included to prevent reflections at $976 \mathrm{~nm}$ and $1064 \mathrm{~nm}$.

When the mode operation changes to pulsed regime the scheme varies slightly. A signal generator must be added before the pulsed laser seed to modulate the signal. Table 1 shows both the average and the peak power delivered by the pulsed laser seed, which depend on the modulation format. The average and the peak power are related to the equation (1), where $\tau$ represents the pulse durations in $s$ and $f$ the repetition rate expressed in $\mathrm{Hz}$. Fig. (2) shows the used modulation signal format.

$P_{\text {peak }}=\frac{P_{\text {average }}(\mathrm{mW})}{f \cdot \tau}$

Table 1. Power Delivered by Pulsed Seed Laser

\begin{tabular}{|c|c|c|c|c|}
\hline Repetition rate, $\boldsymbol{f}(\boldsymbol{k H z})$ & $\mathbf{2 0}$ & $\mathbf{5 0}$ & $\mathbf{1 0 0}$ & $\mathbf{1 0 0}$ \\
\hline \hline Pulse duration, $\tau(n s)$ & 20 & 20 & 20 & 100 \\
\hline $\mathrm{P}_{\text {average }}(d B m)$ & $-10,35$ & $-6,37$ & $-3,32$ & 2,46 \\
\hline $\mathrm{P}_{\text {peak }}(m W)$ & 230,6 & 230,7 & 232,8 & 176,2 \\
\hline
\end{tabular}

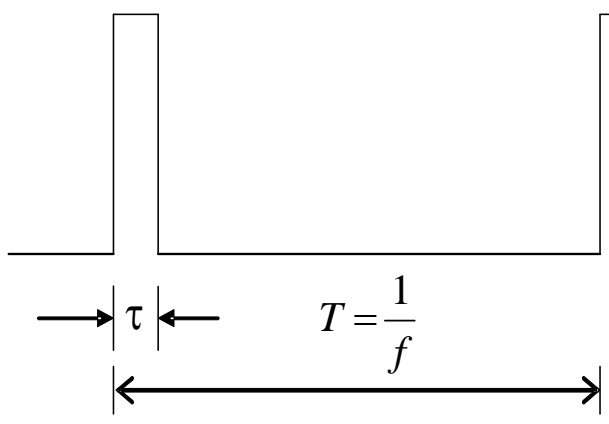

Fig. (2). Modulation signal.

Two different kinds of single cladding YDFs have been used in this experimental set-up. Both are single-mode, single-clad fibers with high Ytterbium concentration. Thus they are suitable for applications where high efficiency and very short lengths are critical. The first YDF is from Nufern, which shows a core absorption about $250 \mathrm{~dB} / \mathrm{m}$ near a wavelength of $975 \mathrm{~nm}$, a core diameter of $6 \mu \mathrm{m}$ and a numerical aperture of 0.11 . The second type is from Pirelli with a core absorption of about $1500 \mathrm{~dB} / \mathrm{m}$ near $975 \mathrm{~nm}$. The great difference in the core absorption between the two fibers is due to the different Ytterbium concentrations. In fact, the Pirelli's fiber dopant concentration is three times higher than Nufern's one. 
All the output powers measured have been obtained using an ASE filter whose bandwidth is $10 \mathrm{~nm}$. When CW regime is used the ASE has not a great influence, whereas in pulsed regime the ASE contribution is remarkable and it must be taken into account.

\section{RESULTS}

The YDFL behaviour has been studied in depth for both kinds of YDFs. For each stage, the output power, the signal and the ASE spectrum have been taken into account to optimize the performance of the laser.

Since the used YDFs present a high doping concentration, the cut-back method has been used to characterize them. The absorption of the fibers is estimated as the difference between the power measured by the optical spectrum analyzer (OSA) directly from a broadband white light source and the power measured in the OSA when the white light goes through the YDF.

The measured absorption for the Pirelli fiber is $809 \mathrm{~dB} / \mathrm{m}$ by using a length of $1 \mathrm{~cm}$ instead of $1500 \mathrm{~dB} / \mathrm{m}$, which is the value that appears in the datasheet. The Nufern fiber absorption is $294 \mathrm{~dB} / \mathrm{m}$ calculated for $4 \mathrm{~cm}$ long fiber. To determine experimentally the absorption of a doped fiber becomes a difficult task when this absorption value is very high. For this reason, the measured absorption of the Pirelli fiber is quite different from the value provided by datasheet. On the contrary, the measured absorption for the Nufern fiber, whose value is smaller than the Pirelli one, is very similar to the datasheet value. Fig. (3) shows the measured spectra of the two analyzed fibers. The utilized OSA parameters are: span from $750 \mathrm{~nm}$ to $1200 \mathrm{~nm}$; highest sensitivity and a resolution bandwidth of $1 \mathrm{~nm}$.

At quick glance, it can be noticed that in Fig. (3a) there is a section in the spectrum with negative losses between 1020 $\mathrm{nm}$ to $1200 \mathrm{~nm}$; Fig. (3b) shows a similar behaviour in two different fragments of the spectrum, the first between 750 $\mathrm{nm}$ to $870 \mathrm{~nm}$, and the second one between $1000 \mathrm{~nm}$ to 1200 $\mathrm{nm}$. This can be explained considering that the white light works like a broadband pump source, although its power is very low, and thus the YDF has an active behaviour in some spectral zones instead of being passive. The higher the concentration of Ytterbium is, more active is its behaviour, even when the input power is very low. This phenomenon makes

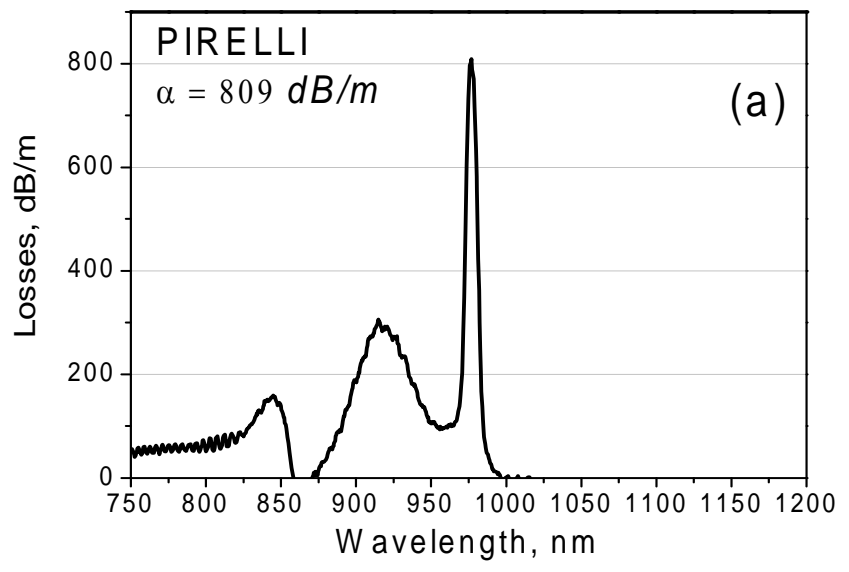

Fig. (3). Absorption spectrum of: (a) Pirelli fiber; (b) Nufern fiber. actually difficult to characterize YDFs with a high Ytterbium concentration.

\subsection{Ytterbium Doped Fiber Laser in CW Regime}

Table 2 shows the output power of the first stage when the Pirelli YDF length varies from $312 \mathrm{~cm}$ to $10 \mathrm{~cm}$ and the seed and pump laser intensities are set at $200 \mathrm{~mW}$ and 300 $\mathrm{mW}$, respectively. The measured maximum output power is $24.8 \mathrm{dBm}$ when the length is $21 \mathrm{~cm}$. However it is remarkable that there are not major differences between the highest

Table 2. Measured Output Power of the First Stage using Pirelli Fiber

\begin{tabular}{|c|c|c|}
\hline Length $(\mathbf{c m})$ & Power $(\boldsymbol{m} \boldsymbol{W})$ & Power $(\mathbf{d B m})$ \\
\hline \hline 312 & 283.8 & 24.53 \\
\hline 150 & 266.7 & 24.26 \\
\hline 100 & 266.7 & 24.26 \\
\hline 50 & 247.7 & 23.94 \\
\hline 37 & 295.8 & 24.71 \\
\hline 30.5 & 261.8 & 24.18 \\
\hline $\mathbf{2 1}$ & $\mathbf{3 0 2 . 0}$ & $\mathbf{2 4 . 8}$ \\
\hline 15.5 & 269.2 & 24.3 \\
\hline 10 & 277.3 & 24.43 \\
\hline
\end{tabular}

and the minimum power, just around $1 \mathrm{~dB}$, even if the fibers length varies considerably. The reason of this behaviour can be found by observing the Ytterbium absorption and emission cross-section profile [6]. At the signal wavelength, that is $1064 \mathrm{~nm}$, the absorption cross-section is very low, thus although the length of the fiber is longer than the optimum one, the part of the unpumped fiber does not introduce remarkable losses. Fig. (4) shows that the measured values are very similar to the simulation results obtained by using the "reservoir" model of the amplifier, already utilized in literature to describe the amplification dynamics [21-23].

Fig. (5) shows the input power of the YDF and its output power in order to calculate the slope-efficiency of the fiber,

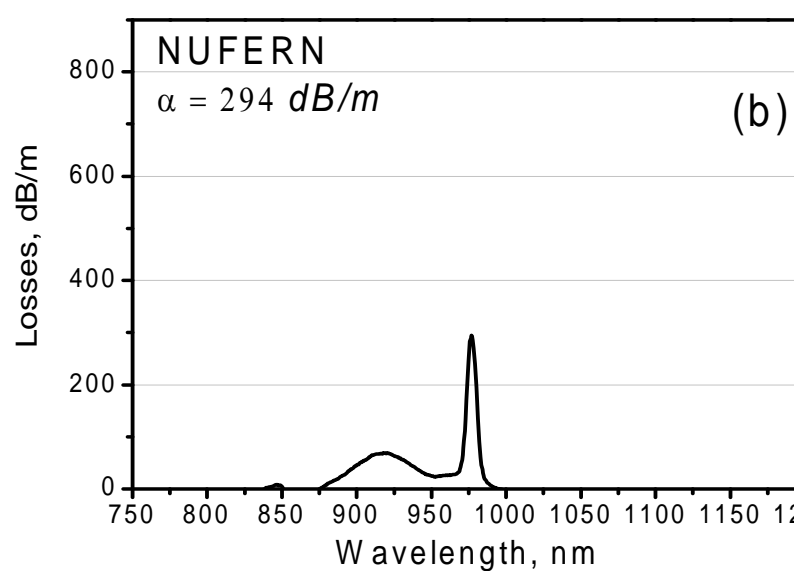




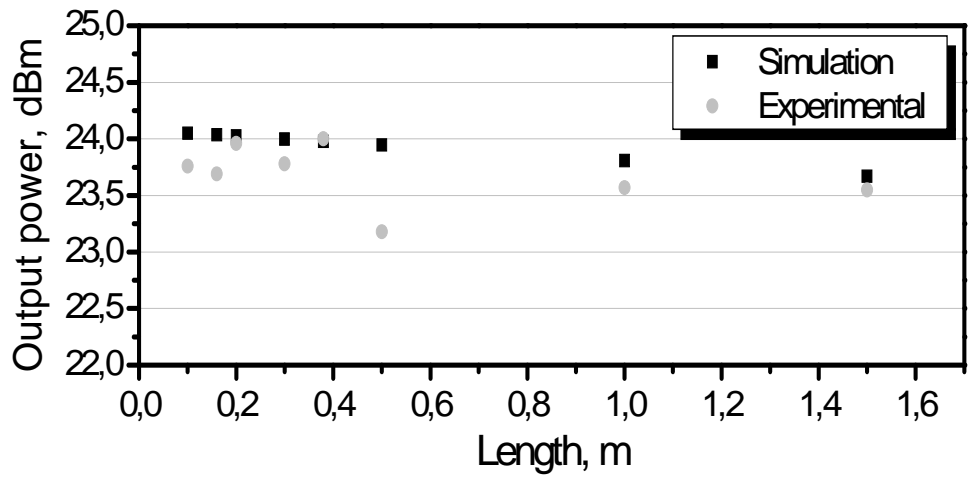

Fig. (4). Comparison of the simulated and experimental values of the output power of the first stage.

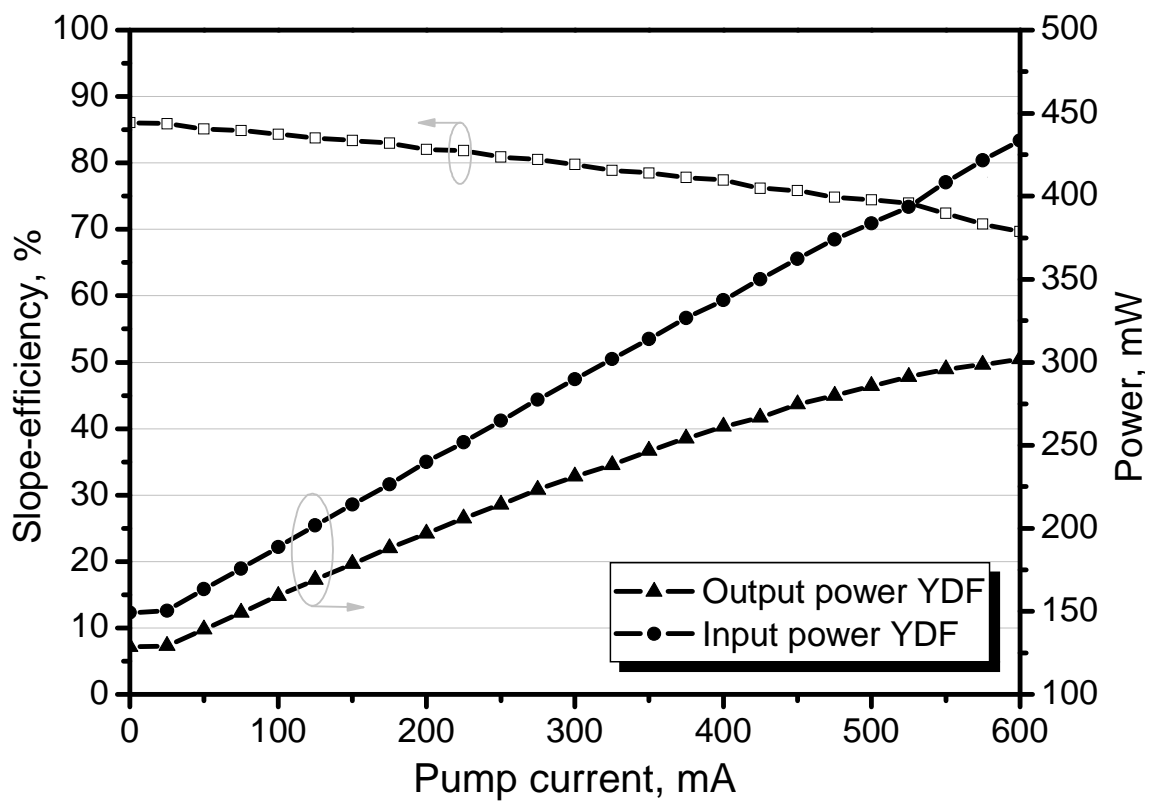

Fig. (5). Slope-efficiency using $21 \mathrm{~cm}$ of Pirelli YDF.

according to (2). In particular, a slope-efficiency value of $69.19 \%$ for the Pirelli YDF has been experimentally determined.

Slope - efficiency $=\frac{\text { OutputPowerYDF }}{\text { TotalPowerInputYDF }}$

Table 3. Maximum Output Power for Different Nufern YDF Lengths at the First Stage

\begin{tabular}{|c|c|}
\hline Length $(\boldsymbol{c m})$ & Power $(\boldsymbol{d B m})$ \\
\hline \hline 101 & 24,34 \\
\hline 86 & 24,39 \\
\hline 70 & 24,47 \\
\hline 62 & 24,63 \\
\hline 58,5 & 24,64 \\
\hline 55,5 & 24,61 \\
\hline
\end{tabular}

An analogous experimental study has been carried out using the Nufern YDF. This fiber, having a different doping concentration, presents a different slope-efficiency respect to the Pirelli fiber. In fact, by averaging out a high number of measurements, it is possible to determine a slope-efficiency for this fiber of about $66 \%$.

The output power has been measured for different lengths from $101 \mathrm{~cm}$ to $55 \mathrm{~cm}$. The seed and pump lasers power is set at $200 \mathrm{~mW}$ and $300 \mathrm{~mW}$, respectively. Table 3 summarizes all the results for the different YDF lengths. The highest power obtained is $24.64 \mathrm{dBm}$ with a YDF length of $58.5 \mathrm{~cm}$. Unsurprisingly, the optimum length using the Nufern YDF is greater than the Pirelli's YDF optimum length, because Nufern's fiber is three times less doped than Pirelli's fiber. The output power reaches with the Pirelli fiber is slightly higher than the output power gets with the Nufern.

Once fixed the optimum YDF lengths for the first stage, the second stage has been evaluated with different YDF lengths in order to get the maximum power. The optimum length for the first stage using the Pirelli YDF is $21 \mathrm{~cm}$ and for the Nufern YDF is $58.5 \mathrm{~cm}$.

Fig. (6a) presents the ASE spectra generated with a pump power of the second stage fixed to $500 \mathrm{~mW}$ and the first stage switched off for three different lengths. The grey continuous line belongs to $11 \mathrm{~cm}$ of fiber. It is an insufficient length and thus the generated $\mathrm{ASE}$ is too low and the majority of the pump is not absorbed by the Ytterbium ions. 


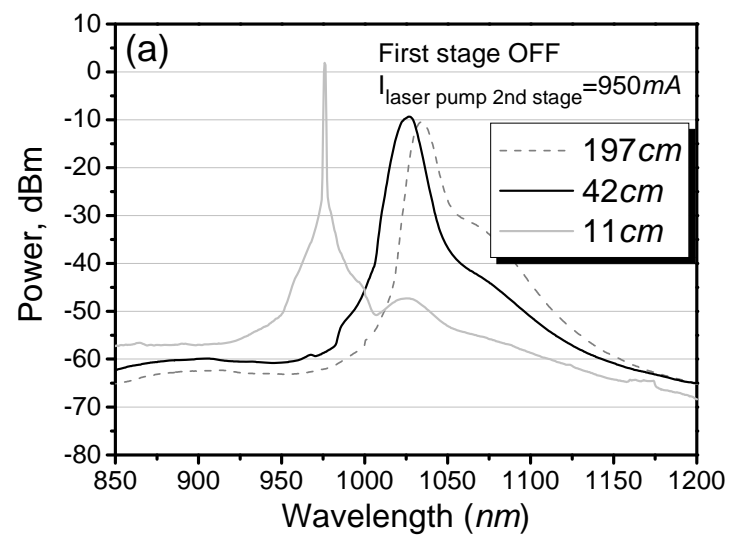

Fig. (6). (a) ASE spectrum; (b) Detail of the ASE spectrum.

In fact, a power peak at the wavelength of $976 \mathrm{~nm}$ can be observed, corresponding to the pump laser emission. When the YDF length increases, the entire pump is absorbed, and a peak of ASE appears near $1030 \mathrm{~nm}$.

Fig. (6b) shows the ASE spectra generated around 1030 $\mathrm{nm}$. It can be noticed that the peak ASE wavelength shifts towards a higher wavelength when the fiber length increases progressively. This is a direct consequence of the unpumped YDF, which behaves like an active ASE filter, shifting the ASE spectrum toward higher wavelengths.

Fig. (7a) shows the signal spectrum with the first stage switched on for different YDF lengths. The seed and pump laser powers are set at 200 and $300 \mathrm{~mW}$ respectively, and the pump laser of the second stage fixed to $500 \mathrm{~mW}$. In order to measure the signal spectra, an attenuator of $20 \mathrm{~dB}$ is used to avoid damages at the OSA detector. The spectrum shapes are very similar around the wavelength corresponding to the emitted signal; however when the fiber is too short, the pump at $975 \mathrm{~nm}$ is not totally absorbed and a residual peak appears at this wavelength.

Table 4 sums up the output power of the laser for different lengths of YDF. The maximum output power is $27.13 \mathrm{dBm}$ using $42 \mathrm{~cm}$ of Pirelli's fiber. The highest laser output power is reached with the Pirelli YDF, since by using

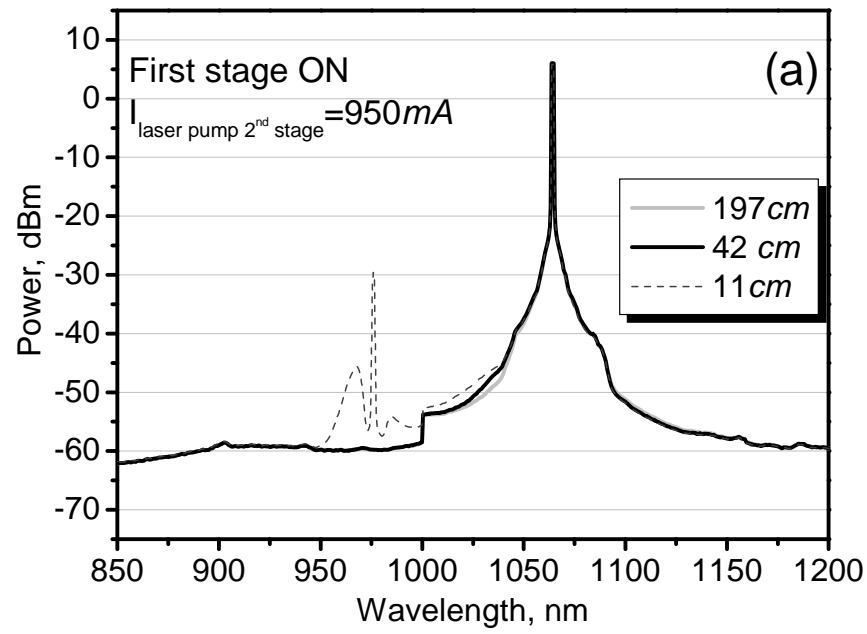

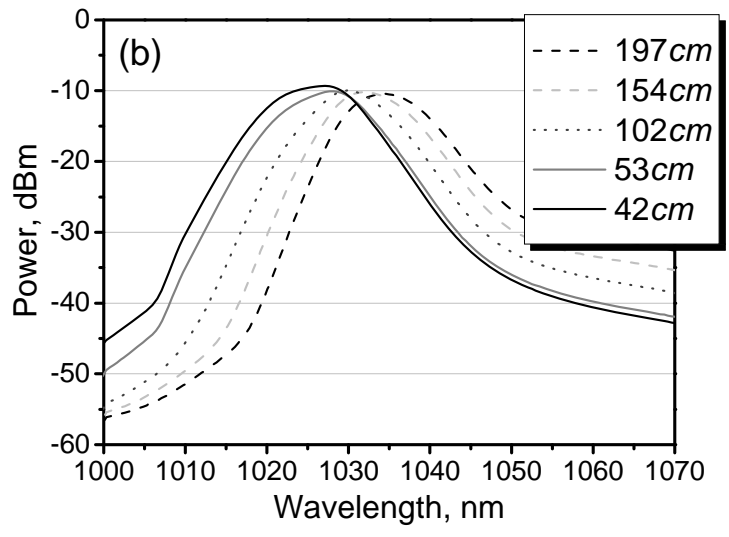

a $70 \mathrm{~cm}$ long YDF by Nufern the maximum output power is $26.39 \mathrm{dBm}$

Table 4. Output Power for Different Lengths of YDF: (a) Pirelli; (b) Nufern

\begin{tabular}{|c|c|}
\hline \multicolumn{2}{|c|}{ (a) } \\
\hline Length (cm) & Power (dBm) \\
\hline 11 & 26.93 \\
\hline 19 & 26.95 \\
\hline 29 & 26.96 \\
\hline 34.5 & 27.07 \\
\hline $\mathbf{4 2}$ & $\mathbf{2 7 . 1 3}$ \\
\hline 53 & 27.03 \\
\hline 102 & 26.97 \\
\hline 154 & 27.01 \\
\hline 197 & 26.97 \\
\hline
\end{tabular}

Notice that the second stage output power of Pirelli YDF has a perfect linear fit in all its range, as shown in Fig. (7b).

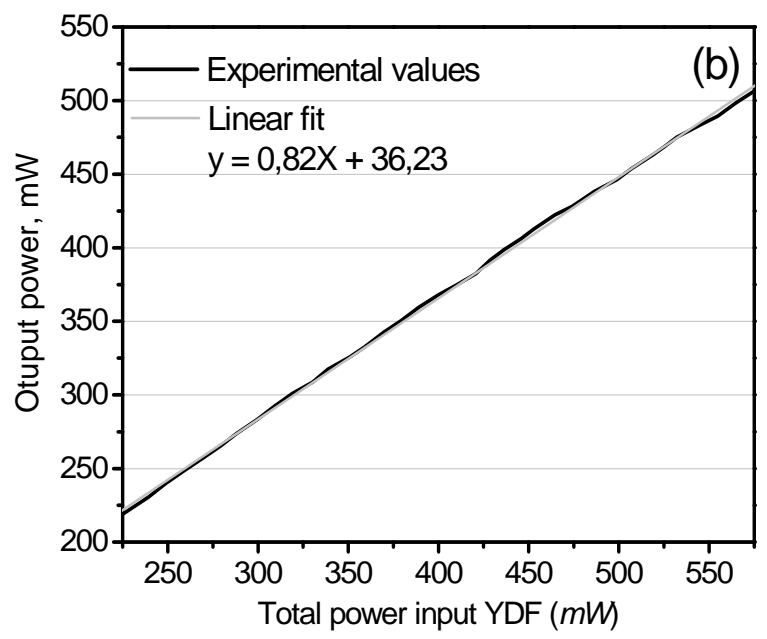

Fig. (7). (a) Signal spectrum; (b) Output power and linear fit with Pirelli YDF length fixed at $42 \mathrm{~cm}$. 
The YDF length is a pivotal factor to optimize because YDFs are very expensive and thus the price of the final laser could increase significantly. On the other hand, the fiber length is a fundamental parameter for the evaluation of the impact of the nonlinear effects on the final fiber laser performances.

\subsection{Ytterbium Doped Fiber Laser in Pulsed Regime}

A study of the first stage YDFL using a pulsed seed laser has been also carried out. The goal of this section is to achieve the maximum peak power. Four different lengths of Pirelli YDF have been employed: $2.99 \mathrm{~m}, 2.49 \mathrm{~m}, 1.96 \mathrm{~m}$ and $1.75 \mathrm{~m}$.

The highest peak power has been obtained with a $1.96 \mathrm{~m}$ long YDF, by using a modulation signal with the lowest pulse duration $(20 \mathrm{~ns})$ and a repetition rate of $20 \mathrm{kHz}$. The pump power is fixed at $300 \mathrm{~mW}$. With these parameters, the obtained mean power is $10.81 \mathrm{dBm}$ and the peak power reaches $30 \mathrm{~W}$. Table 5 summarizes the average and peak power obtained for the optimum fiber length for different pulse durations and repetition rates. When the pulse duration keeps at the same value, that is $20 \mathrm{~ns}$, and the repetition rate increases, the average power rises, but the peak power slightly decreases.

Table 5. Average and Peak Power with a $1.96 m$ Long YDF

\begin{tabular}{|c|c|c|c|c|}
\hline & \multicolumn{2}{|c|}{ Average power } & \multicolumn{2}{c|}{ Peak power } \\
\hline & $\mathbf{( d B m})$ & $\mathbf{( m W})$ & $\mathbf{( d B m})$ & $\mathbf{( m W )}$ \\
\hline \hline $\boldsymbol{\tau}=\mathbf{2 0 n s ;} ; \mathbf{f}=\mathbf{2 0 k H z}:$ & 10.81 & 12.05 & $\mathbf{4 4 . 7 9}$ & $\mathbf{3 0 1 2 5 . 9 0}$ \\
\hline $\boldsymbol{\tau}=\mathbf{2 0 n s ;} ; \mathbf{f}=\mathbf{5 0 k H z}:$ & 13.09 & 20.37 & 43.09 & 20370.42 \\
\hline $\boldsymbol{\tau}=\mathbf{2 0 n s} ; \mathbf{f}=\mathbf{1 0 0 k H z}:$ & 14.58 & 28.71 & 41.57 & 14353.90 \\
\hline $\boldsymbol{\tau}=\mathbf{1 0 0 n s ;} ; \mathbf{f}=\mathbf{1 0 0 k H z}:$ & 16.84 & 48.31 & 36.84 & 4830.59 \\
\hline
\end{tabular}

Fig. (8) presents the peak power for the different lengths of YDF, fixed the pulsed regime with a pulse duration of 20 ns and repetition rate of $20 \mathrm{kHz}$. The obtained values ranged from $25 \mathrm{~W}$ to $30 \mathrm{~W}$. To calculate the peak power, the average power has been measured by using a power meter and an ASE filter. By means of equation (1) it is easy to estimate the peak power from the average one.

As it has been mentioned previously, in the pulsed regime the ASE noise has a great influence in the measured output power. This behaviour is easily explainable considering that during the most part of the repetition period the pump is on and the signal is switch off and so, during these intervals there is no signal to generate stimulated emission and thus the ASE noise appears. Fig. (9) corroborates experimentally the previous explanation. It plots the signal spectrum when the length is fixed to $1.96 \mathrm{~m}$, the pulse duration is $20 \mathrm{~ns}$ and the repetition rate varies. When the repetition rate is low, the ASE contribution is more evident because the signal is less present and the pump is exploited to generate ASE. Finally, Fig. (10) points out the obtained output peak power for the best case, that is when the YDF length (1) equals to $1.96 \mathrm{~m}, \tau=20 \mathrm{~ns}$ and $\mathrm{f}=20 \mathrm{KHz}$. The figure shows the measured powers both with and without the
ASE filter. The maximum difference is around $7 \mathrm{~W}$ that is a non-negligible amount of power.

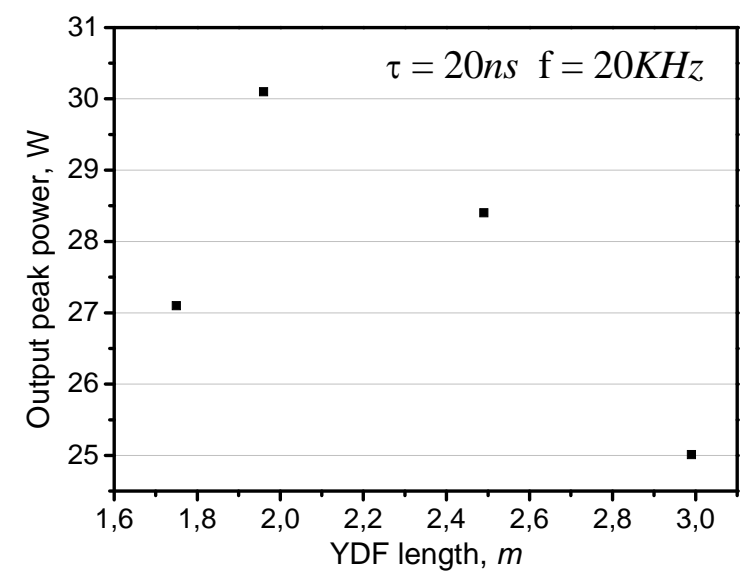

Fig. (8). Peak power for different lengths of YDF.

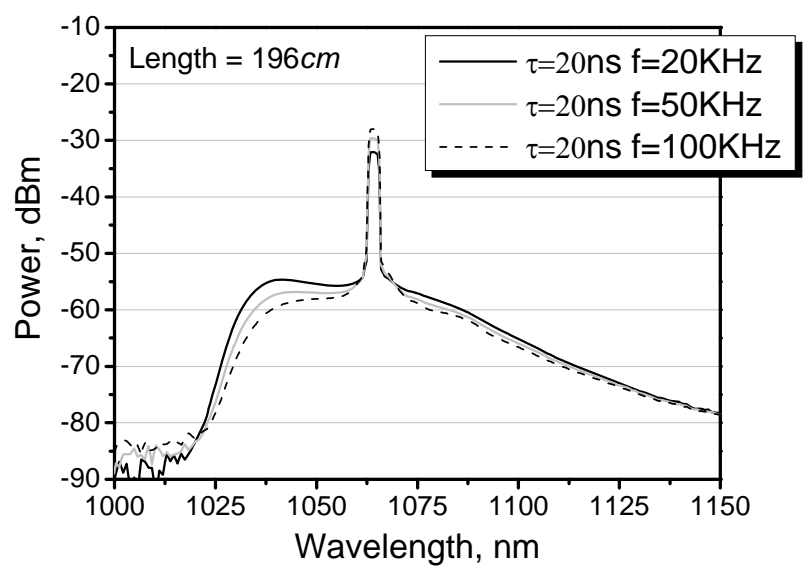

Fig. (9). Signal spectrum for different repetition rates.

Fig. (11) shows the signal spectra obtained by the OSA. A $40 \mathrm{~dB}$ attenuator is used in order not to damage the OSA with a maximum input power around $10 \mathrm{dBm}$. Notice that the peak power of ASE generated around $1030 \mathrm{~nm}$ increases when the YDF length decreases. In fact, the YDF acts as ASE filter when the length is greater than the optimum length.

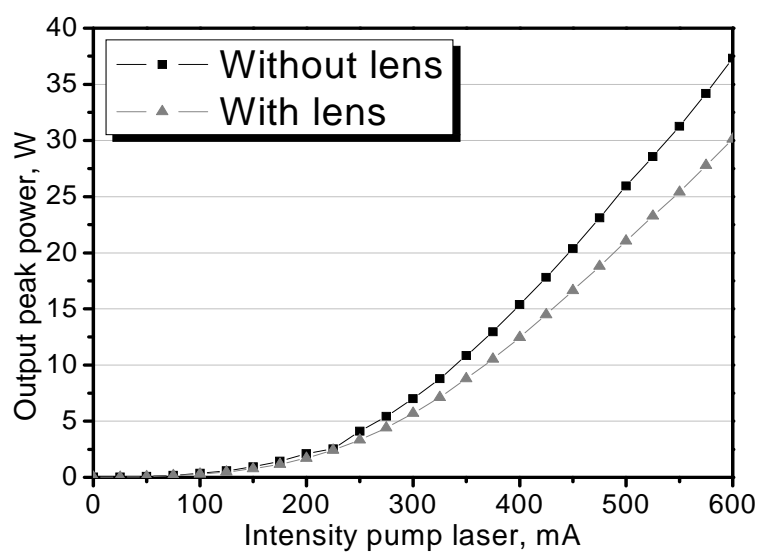

Fig. (10). Peak power with and without ASE filter. 


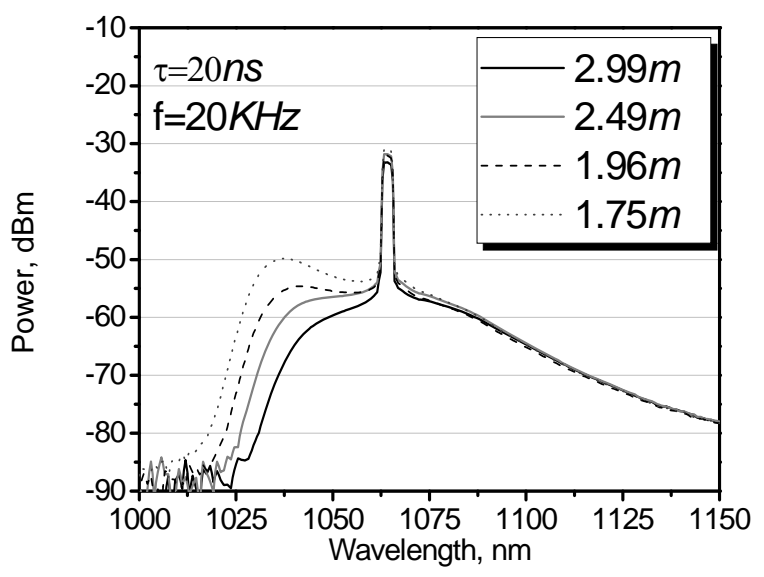

Fig. (11). Signal spectrum for different lengths.

Comparing Figs. (7a, 9 and 11), the differences between the shape signal spectrum in $\mathrm{CW}$ and pulsed regime can be analyzed. It is evident that in CW regime the ASE in the signal spectrum is much lower than in the pulsed regime.

The presented first stage of the Pirelli YDFL in pulsed regime could be used as a pre-amplifier for a second stage, that is, it would be a master oscillator power amplifier (MOPA) of a second stage. This could be composed by a double clad fiber [10] in order to scale the output power of the laser and get a higher power laser. This experiment is currently under development.
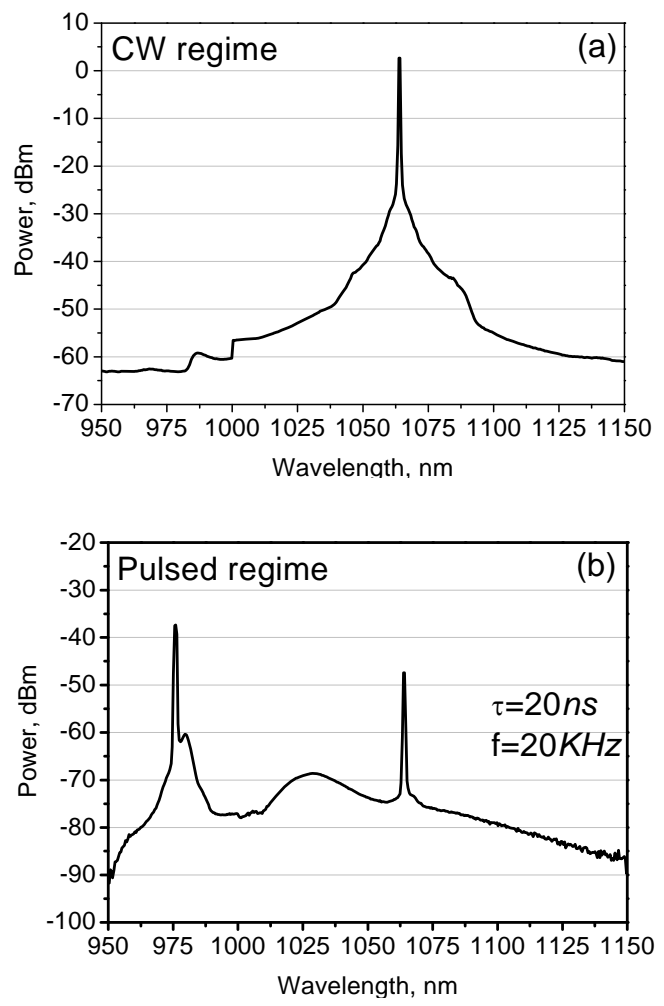

Fig. (12). Signal spectrum with $55 \mathrm{~cm}$ long Nufern YDF: (a) CW regime; (b) pulsed regime.

Finally, Figs. (12a and b) show a comparison of the signal spectrum in $\mathrm{CW}$ and in pulsed regime respectively by using a $55 \mathrm{~cm}$ long Nufern fiber. Notice that the different power scale of the two figures is due to the use of different attenuation values of the attenuators located at the input of the OSA. Moreover, in CW regime (Fig. 12a) the pump is totally absorbed, whereas in the pulsed regime (Fig. 12b) the pump power is not completely absorbed. This suggests that the optimization of the YDF length is strictly dependent on the operation regime of the final fiber laser. In conclusion, the configuration of the laser is strictly dependent on the final industrial application, so that it is not possible to design a general purpose fiber laser.

\section{DISCUSSION AND CONCLUSIONS}

A two-stage YDFL has been presented. Two kinds of YDFs with different concentration of Ytterbium ions have been used. The proposed laser is made up of two stages. Each stage has been experimentally studied with different YDF lengths in order to find the optimum output power. The signal and ASE spectra have been also shown. The first stage has been also studied in both $\mathrm{CW}$ and pulsed regime. A comparison between both regimes has been carried out.

\section{ACKNOWLEDGEMENTS}

This work was supported by the Spanish Government projects TEC2007-67987-C02 and TEC2010-20224-C02-01, the European COST action-299 and the Spain-Italy Cooperation Project "Development of new all-fiber lasers for telecom, sensors and industrial applications".

\section{CONFLICT OF INTEREST}

None declared.

\section{REFERENCES}

[1] Dominic V, MacCormack S, Waarts R, et al. $110 \mathrm{~W}$ fibre laser. Electron Lett 1999; 35(14): 1158-1160.

[2] Limpert J, Liem A, Zellmer H, TuNnermann A. $500 \mathrm{~W}$ continuouswave fibre laser with excellent beam quality. Electron Lett 2003; 39(8): 645-647.

[3] Jeung Y, Sahu JK, Payne DN, Nilsson J. Ytterbium-doped largecore fibre laser with $1 \mathrm{~kW}$ of continuous-wave output power. Electron Lett 2004; 40(8): 470-472.

[4] Jeong Y, Sahu JK, Payne DN, Nilsson J. Ytterbium-doped largecore fiber laser with $1.36 \mathrm{~kW}$ continuous-wave output power. Opt Express 2004; 12(25): 6088-6092.

[5] Serelli S, Poli F. Doped fiber lasers: From telecom to industrial applications. Proceedings of the 10th Anniversary International Conference on Transparent Optical Networks; June 2008; Athens, Greece; 2008.

[6] Paschotta R, Nilsson J, Tropper AC, Hanna DC. Ytterbium-doped fiber amplifiers. IEEE J Quant Electron 1997; 33(7): 1049-1056.

[7] Kurkov AS. Oscillation spectral range of Yb-doped fiber lasers. Laser Phy Lett 2007; 4(2): 93-102.

[8] Limpert J, Röser F, Klingebiel S, et al. The rising power of fiber lasers and amplifiers. IEEE J Selected Topics Quantum Electron 2007; 13(3): 537-544.

[9] Wang Y. Optimization of pulse amplification in ytterbium-doped double-clad fiber amplifiers. J Lightwave Technol 2005; 23(6): 2139-2147.

[10] Filippov V, Chamorovskii Y, Kerttula J, Golant K, Pessa M, Okhotnikov OG. Double clad tapered fiber for high power applications. Opt Express 2008; 16(3): 1929-1944.

[11] Efficiency of active double-clad fiber pumping in different types of fibers. 2008 10th Anniversary International Conference on Transparent Optical Networks; June 2008; Athens, Greece; 2008. 
[12] Liu A, Ueda K. The absorption characteristics of circular, offset, and rectangular double-clad fibers. Opt Commun 1996; 132(5-6): 511-518.

[13] Broderick NGR, Offerhaus HL, Richardson DJ, Sammut RA, Caplen J, Dong L. Large mode area fibers for high power applications. Opt Fiber Technol 1999; 5(2): 185-196.

[14] Tsuchida Y, Saitoh K, Koshiba M. Design of single-moded holey fibers with large-mode-area and low bending losses: The significance of the ring-core region. Opt Express 2007; 15(4): 17941803.

[15] Russell PSJ. Photonic-crystal fibers. J Lightwave Technol 2006; 24(12): 4729-4749.

[16] Poli F, Cucinotta A, Passaro D, Selleri S, Lægsgaard J, Broeng J. Single-mode regime in large-mode-area rare-earth-doped rod-type PCFs. IEEE J Selected Topics Quantum Electron 2009; 15(1): 5460.

[17] Akowuah EK, Ademgil H, Haxha S, AbdelMalek F. An endlessly single-mode photonic crystal fiber with low chromatic dispersion, and bend and rotational insensitivity. J Lightwave Technol 2009; 27(17): 3940-3947.

[18] Furusawa K, Malinowski A, Price JHV, et al. Cladding pumped ytterbium-doped fiber laser with holey inner and outer cladding. Opt Express 2001; 9(13): 714-720.

[19] Chen ZJ, Grudinin AB, Porta J, Minelly JD. Enhanced Q switching in double-clad fiber lasers. Opt Lett 1998; 23(6): 454-456.

[20] Liem A, Limpert J, Zellmer H, TuNnermann A. 100-W singlefrequency master-oscillator fiber power amplifier. Opt Lett 2003; 28(17): 1537-1539.

[21] Bononi A, Rusch LA. Doped-fiber amplifier dynamics: A system perspective. J Lightwave Technol 1998; 16(5): 945-956.

[22] Bononi A, Papararo M, Fuochi M. Transient gain dynamics in saturated Raman amplifiers. Opt Fiber Technol 2004; 10(1): 91123.

[23] Mathlouthi W, Lemieux P, Salsi M, Vannucci A, Bononi A, Rusch LA. Fast and efficient dynamic WDM semiconductor optical amplifier model. J Lightwave Technol 2006; 24(11): 4353-4365.

(C) Fernandez-Vallejo et al.; Licensee Bentham Open.

This is an open access article licensed under the terms of the Creative Commons Attribution Non-Commercial License (http://creativecommons.org/ licenses/by-nc/3.0/), which permits unrestricted, non-commercial use, distribution and reproduction in any medium, provided the work is properly cited. 